\title{
A New Formalism for Classifying Spin-Orbit Systems Using Tools Distilled from the Theory of Bundles
}

\author{
K. Heinemann \\ Department of Mathematics and Statistics, University of New Mexico, \\ Albuquerque, New Mexico 87131, USA \\ heineman@math.unm.edu \\ D. P. Barber \\ Deutsches Elektronen-Synchrotron, DESY, \\ 22607 Hamburg, Germany \\ mpybar@mail.desy.de \\ J. A. Ellison \\ Department of Mathematics and Statistics, University of New Mexico, \\ Albuquerque, New Mexico 87131, USA \\ ellison@math.unm.edu \\ M. Vogt \\ Deutsches Elektronen-Synchrotron, DESY, \\ 22607 Hamburg, Germany \\ vogtm@mail.desy.de \\ Published 29 February 2016
}

\begin{abstract}
We give an informal summary of ongoing work which uses tools distilled from the theory of fibre bundles to classify and connect invariant fields associated with spin motion in storage rings. We mention four major theorems. One ties invariant fields with the notion of normal form, the second allows comparison of different invariant fields and the two others tie the existence of invariant fields to the existence of certain invariant sets. We explain how the theorems apply to the spin dynamics of spin- $1 / 2$ and spin- 1 particles. Our approach elegantly unifies the spin-vector dynamics from the T-BMT equation with the spin-tensor dynamics and other dynamics and suggests an avenue for addressing the question of the existence of the invariant spin field.
\end{abstract}

This is an Open Access article published by World Scientific Publishing Company. It is distributed under the terms of the Creative Commons Attribution 3.0 (CC-BY) License. Further distribution of this work is permitted, provided the original work is properly cited. 


\section{Introduction}

The polarization of a beam of spin-1/2 or spin-1 particles circulating in a storage ring is best systematized in terms of invariant spin fields (ISF's) and invariant polarization-tensor fields (ITF's). They are essential for describing the equilibrium polarization state of proton, deuteron and electron beams.

We have already treated the concept of the ISF in depth in Ref. 1. Numerical evidence indicates that ISF's can be rather complex entities. Moreover the question of existence, although trivial in some simple cases, e.g., on orbital resonance, is up to this day, unsolved and, as evidenced by use of stroboscopic averaging ${ }^{2}$ model situations can occur off orbital resonance where ISF's might not exist. In other words there is no good understanding of the conditions under which the ISF exists off orbital resonance. Nevertheless we believe that practically relevant spin-orbit systems which have no such ISF are "rare". We call this the "ISF-conjecture".3-5 This has motivated us to extend our studies using a new approach, namely with tools from Dynamical-Systems theory developed in the 1980s by R. Zimmer, R. Feres and others ${ }^{6-8}$ and following previous work by one of us $(\mathrm{KH})$ in his $\mathrm{PhD}$ thesis. ${ }^{9}$ In particular, this approach enables us to generate, classify and study new and old invariant fields and it provides an avenue for addressing the question of the existence of ISF's. Since we work in the framework of topological dynamical systems, all functions of interest are continuous, in particular the ISF.

We have proved several major theorems, among which are the following four. They are the Normal Form Theorem, tying invariant fields with the notion of normal form, the Decomposition Theorem, allowing comparison of different invariant fields, the Invariant Reduction Theorem, giving new insights into the question of the existence of invariant fields and which is supplemented by the Cross Section Theorem. It turns out that the well-established notions ${ }^{1}$ of invariant frame field and uniform invariant frame field are generalized by the normal form concept whereas the well-established notions of ISF and ITF are generalized to the invariant $(E, l)$ fields to be defined later. With the flexibility in the choice of $(E, l)$, we have a unified way to study, for example, the dynamics of spin- $1 / 2$ and spin- 1 particles and the density matrices of the corresponding particle bunches.

Our formalism, which we call the Technique of Association (ToA), has its origins in Bundle Theory so that our methods can be compared with those used in YangMills Theory. We thus open a significant new area of research in our field by bringing in techniques from Bundle Theory used hitherto in very different research areas. We believe that all four of these theorems are important. Furthermore, we come to a new view of the ISF via its link to the existence of a certain invariant set.

Owing to the page limit, this account in necessarily just an informal summary of this work with which we wish to increase its accessibility for accelerator physicists. We hope that the reader will be motivated to look at the complete and rigorous accounts of the mathematics in Refs. $3-5$ where important notation and terminology are also explained. 


\section{The Basic Equations}

We assume that the reader is familiar with the T-BMT equation for spin motion, the invariant spin field (ISF) and the amplitude-dependent spin tune (ADST). ${ }^{1}$

In contrast to Ref. 1, we now work in terms of 1-turn maps for the orbital and spin motion. The 1-turn spin map from the T-BMT equation starting at azimuth $\theta_{0}$ with orbital phases $\phi\left(\theta_{0}\right)$ and with amplitudes $J$ is

$$
S\left(\theta_{0}+2 \pi\right)=A\left(\theta_{0}, \phi\left(\theta_{0}\right), J\right) S\left(\theta_{0}\right)
$$

where $S$ is the column matrix of the three components of the spin vector and where the 1 -turn spin map $A(\theta, \phi, J)$ is a real orthogonal $3 \times 3$ matrix of unit determinant, i.e., an element of $S O(3)$, and it is a function of $(\theta, \phi, J), 2 \pi$-periodic in $\theta$ and the components of $\phi$. Over one turn, the position $\phi$ on the $d$-torus $\mathbb{T}^{d}$ is transformed to $j_{J}(\phi)$. Usually $j_{J}(\phi)=\phi+2 \pi \omega(J)$ where $\omega(J)$ is the set of orbital tunes. Normally $d=1,2$ or 3 . We ignore the ambiguous and miniscule Stern-Gerlach forces. For our integrable orbital motion $J$ is a constant parameter which we now suppress.

\section{The Invariant Spin and Tensor Fields}

As explained in Refs. 1 and 5 the maximum attainable vector polarization of a beam of particles of arbitrary spin is expressed in terms of the invariant spin field (ISF). An ISF, $f_{v}(\theta, \phi)$, being a spin field, evolves as a consequence of the T-BMT equation so that after one turn $f_{v}(\theta+2 \pi, j(\phi))=A(\theta, \phi) f_{v}(\theta, \phi)$. By definition $\left|f_{v}\right|=1$ and $f_{v}$ is $2 \pi$-periodic in $\theta$, i.e., $f_{v}(\theta+2 \pi, \phi)=f_{v}(\theta, \phi)$. Then $f_{v}(\theta, j(\phi))=A(\theta, \phi) f_{v}(\theta, \phi)$. Note that in Refs. 1 and 10 and in most other literature on ISF's, the ISF is written as $\hat{n}(\theta, \phi)$.

In Refs. 3-5 and this work we "sample" the particle and spin motion once per turn at some fixed azimuth $\theta_{0}$. So we suppress the $\theta_{0}$ and write $f_{v}(j(\phi))=$ $A(\phi) f_{v}(\phi)$.

For deuterons (spin-1) a full description of the equilibrium polarization state of a beam involves the invariant polarization-tensor field (ITF), $f_{t}$. This is a $3 \times 3$, real, symmetric, traceless, field on $\mathbb{T}^{d}$ which we also take to be continuous in $\phi$. The invariance of $f_{t}$ over one turn gives: $f_{t}(j(\phi))=A(\phi) f_{t}(\phi) A(\phi)^{T}$. As shown in

Ref. 10 one may parametrize $f_{t}$ as $f_{t}= \pm \sqrt{\frac{3}{2}}\left\{\hat{n} \hat{n}^{T}-\frac{1}{3} I\right\}\left(\equiv \pm \sqrt{\frac{3}{2}}\left\{f_{v} f_{v}^{T}-\frac{1}{3} I\right\}\right)$.

\section{A Unified Representation of the Transformations}

We can encompass the varieties of "spin" dynamics in $S O(3)$-spaces, $(E, l)$, where $E$ is a topological space and $l$ is a continuous $S O(3)$-action on $E$, i.e., $l: S O(3) \times E \rightarrow$ $E$ is continuous and $l(I ; x)=x$ and $l\left(r_{1} r_{2} ; x\right)=l\left(r_{1} ; l\left(r_{2} ; x\right)\right)$ with $x \in E$.

For spin vectors we have $(E, l)=\left(\mathbb{R}^{3}, l_{\mathrm{v}}\right)$ where $l_{\mathrm{v}}(r ; S):=r S$ and $r \in S O(3)$. For a spin tensor $M$ we invoke $\left(E_{\mathrm{t}}, l_{\mathrm{t}}\right)$ where $l_{\mathrm{t}}(r ; M):=r M r^{\mathrm{t}}$ and $M \in E_{t}, r \in$ $S O(3)$. These are examples of the flexibility in the choice of $(E, l)$ mentioned in the Introduction. 
In this setting, over one turn, a field $f$ becomes the field $f^{\prime}$ where $f^{\prime}(\phi):=$ $l\left(A\left(j^{-1}(\phi)\right) ; f\left(j^{-1}(\phi)\right)\right)$ or: $f \mapsto f^{\prime}=l\left(A \circ j^{-1} ; f \circ j^{-1}\right)$. By definition, an invariant field maps into itself (the whole field) i.e., $f^{\prime}=f$. Then invariance implies that $f(j(\phi))=l(A(\phi) ; f(\phi))$. This, of course, reproduces the definitions of invariance of the ISF and the ITF given earlier. Appropriate $S O(3)$-spaces can be introduced for handling the density matrices and other objects related to spin.

With the language of $S O(3)$-spaces we are now in a position to understand the relationship between invariant fields and certain subgroups of $S O(3)$ as well as to explore the relationships between different invariant fields and to classify them. Moreover we can associate invariant fields with certain invariant sets. We call our approach the Technique of Association following its origins in Bundle Theory and in the so-called associated bundles lying behind our structures.

\section{Normal Forms and the Normal Form Theorem (NFT)}

Spin motion can in general look complicated, especially close to spin-orbit resonances. ${ }^{1,5}$ Nevertheless it can often be made to look simple by a proper continuous choice of a coordinate system $T \in \mathcal{C}\left(\mathbb{T}^{d}, S O(3)\right)$ for the spin at each point on the torus. Then we have $A^{\prime}(\phi):=T^{t}(j(\phi)) A(\phi) T(\phi)$ and within $T$ a spin vector $S$ is $S^{\prime}=T^{t}(\phi) S$. If the unit-length third axis of $T$ is the ISF, we call it an invariant frame field (IFF). The motion of $S^{\prime}$ is then a simple precession around the ISF and $A^{\prime}(\phi) \in S O(2)$. Away from orbital resonance, the other two axes of the IFF can usually be chosen so that the rate of precession of $S^{\prime}$ in the IFF is independent of the orbital phases. This rate of precession is the ADST and this leads to the definition of spin-orbit resonance. See Refs. 1 and 5 for details.

In general we can classify the $A^{\prime}$ according to their membership of subgroups $H$ of $S O(3)$ and we then call an $A^{\prime}$ an $H$-normal form.

This brings us to the Normal Form Theorem. We do not state it explicitly here but as explained in Refs. 3-5 the NFT exploits the structures of $S O(3)$-spaces and it shows that an invariant field can be associated with a so-called isotropy group $H$ which gives us a particular $H$-normal form.

Thus the ISF is associated with the subgroup $S O(2)$ of $S O(3)$. Off orbital resonance, the ITF is generically associated with the subgroup $S O(2) \bowtie Z(2)$ of $S O(3)$ where $\bowtie$ denotes a Zappa-Szép product. The Normal Form Theorem shows how the notion of IFF associated with $S O(2)$ can be generalized to apply to other isotropy groups, thereby providing a new view of the IFF, and it also provides a protocol for constructing further invariant $(E, l)$-fields. Moreover, it leads naturally to the Decomposition Theorem.

\section{The Decomposition Theorem (DT)}

Since invariant fields are tied to subgroups of $S O(3)$, these subgroups can be used to classify and relate invariant fields. For this we exploit the Decomposition Theorem. Again, we do not state the theorem, but as explained in Refs. 3-5, it shows how, 
when $\mathbb{T}^{d} \times E$ has been decomposed into certain disjoint invariant sets, the decompositions (the sets) in the same or different $(E, l)$ can be related and classified. In fact we have the powerful result that if the isotropy groups tied to two invariant fields are conjugate, then the two invariant fields are related by a homeomorphism. Moreover, if we know one invariant field, we can use homeomorphisms to construct others.

As an example we can use the relationship between the isotropy groups tied to the ISF and ITF respectively to construct the ITF from the ISF: $f_{t}=$ $\pm \sqrt{\frac{3}{2}}\left\{\hat{n} \hat{n}^{\mathrm{T}}-\frac{1}{3} I\right\}$. In other words we can generate (say) the ITF from the ISF without recourse to physics! - we have a machine to generate non-arbitrary invariant fields. Other examples of such generation can be found in Refs. 3-5.

\section{The Invariant Reduction Theorem (IRT) and the Cross Section Theorem (CST)}

Our next offering is the Invariant Reduction Theorem. ${ }^{3-5}$ In Section 8.7 of Ref. 5 we introduce a 1-turn map $\hat{\mathcal{P}}$ on $\mathcal{E}_{d}:=\mathbb{T}^{d} \times S O(3)$ and a special subset, $\hat{\mathcal{E}}_{d}[f]$ of $\mathcal{E}_{d}$ depending on a field $f$. Then $f$ is an invariant field iff $\hat{\mathcal{E}}_{d}[f]$ is invariant under $\hat{\mathcal{P}}$.

Thus the important question of the existence of an invariant field, and of the ISF in particular, reduces to finding $f$ such that $\hat{\mathcal{E}}_{d}[f]$ is invariant under $\hat{\mathcal{P}}$ and so $\hat{\mathcal{E}}_{d}[f]$ is the tool that we will use.

The CST is even more technical than the previous three theorems and it goes beyond the scope of this short summary. Suffice it to say that the CST deals with so-called cross sections. A cross section is the right-inverse of the natural projection of $\hat{\mathcal{E}}_{d}[f]$ into $\mathbb{T}^{d}$ when $f$ is an invariant field. For example, if $f$ is an ISF then there is a cross section iff there is an IFF whose third column is $f$. Thus cross-sections give new insights into ISF's and ITF's and the CST provides an additional route for examining the question of the existence of the ISF. The reader should consult Refs. 3-5 for details.

\section{Underlying Bundle Theory}

While it has not been necessary above to become immersed in the bundle-theoretic basis of the ToA it is still appropriate to mention it since it supplies a steady flow of ideas. More on the mathematics can be found in Refs. 7, 8 and 11 and in Refs. 3-5 and 9. We can also draw analogies with the way that bundles are used in gauge theories for elementary particles and see how path lifting to parallel-transport motions can reproduce the T-BMT equation.

The "unreduced" principal bundle underlying our formalism is a product principal $S O(3)$-bundle with base space $\mathbb{T}^{d}$. For the "unreduced" principal bundle underlying a gauge theory the base space is a patch of space-time and the fibres are elements of the gauge group. The transformation rule $A^{\prime}(\phi):=T^{t}(j(\phi)) A(\phi) T(\phi)$ has its bundle counterpart in a transformation rule under the $S O(3)$ gaugetransformation group ${ }^{11}$ of the unreduced principal bundle. 
Every $(E, l)$ in the formalism uniquely determines an "associated bundle", relative to the unreduced bundle. In our formalism the invariant $(E, l)$-fields are the nontrivial data of invariant cross sections of associated bundles. This is analogous to the case of gauge theories where the matter fields carry the data of cross sections of associated bundles. With this we have "geometrized" the invariant fields and provided a new view of invariant fields.

\section{Summary and Plans}

We have a new, concise and powerful formalism, based on the concept of $S O(3)$ spaces $(E, l)$, for defining, generalizing, creating and classifying invariant fields in storage rings using tools inspired by Bundle Theory. In fact by their origin in bundle theory, invariant fields and IFF's are rather deep concepts. ${ }^{9}$ Furthermore we have a new tool for studying the question of the existence of invariant $(E, l)$-fields and in particular the existence of the ISF. With no ISF, there can be no equilibrium polarization. We believe that this is the first application of these methods ${ }^{6-8}$ in accelerator physics.

\section{Acknowledgments}

Work has been supported by DOE under DE-FG-99ER41104 and by DESY. We thank Dan T. Abell for help with preparing figures for the talk itself.

\section{References}

1. D.P. Barber, J.A. Ellison and K. Heinemann, Phys. Rev. ST Accel. Beams 7, 124002 (2004).

2. K. Heinemann and G.H. Hoffstaetter, Phys. Rev. E 54 (4), 4240 (1996).

3. K. Heinemann, D.P. Barber, J.A. Ellison and M. Vogt, Proc. 2014 Int. Particle Accelerator Conference, Dresden, Germany, June 2014, JACOW, (2014).

4. K. Heinemann, D.P. Barber, J.A. Ellison and M. Vogt, arXiv:1409.4373v2 (physics.accph, math-ph, math.MP) (2014). Submitted for publication.

5. K. Heinemann, D.P. Barber, J.A. Ellison and M. Vogt arXiv:1501.02747v1 (physics.accph, math-ph, math.MP) (2015). Submitted for publication.

6. R.J. Zimmer, Ergodic theory and the automorphism group of a G-structure, Proc. of a conference in honor of George W. Mackey, Berkeley, USA, 1984. Mathematical Sciences Research Institute Publications, (Springer-Verlag, 1987), p. 247.

7. R. Feres, Dynamical systems and semisimple groups: an introduction, (Cambridge University Press, Cambridge, 1998).

8. R. Feres and A. Katok, Handbook of dynamical systems Vol. 1A. eds. B. Hasselblatt and A. Katok, (North-Holland, Amsterdam, 2002).

9. K. Heinemann, Two Topics in Particle Accelerator Beams, Doctoral Thesis, University of New Mexico (2010).

10. D.P. Barber and M. Vogt, The Invariant Polarisation-Tensor Field for Spin-1 Particles in Storage Rings, Proc. 18th Int. Spin Physics Symposium, Charlottesville, USA, October 2008, (AIP proceedings 1149, 2008).

11. D. Husemoller, Fibre Bundles, 3rd edn. (Springer-Verlag, New York, 1994). 